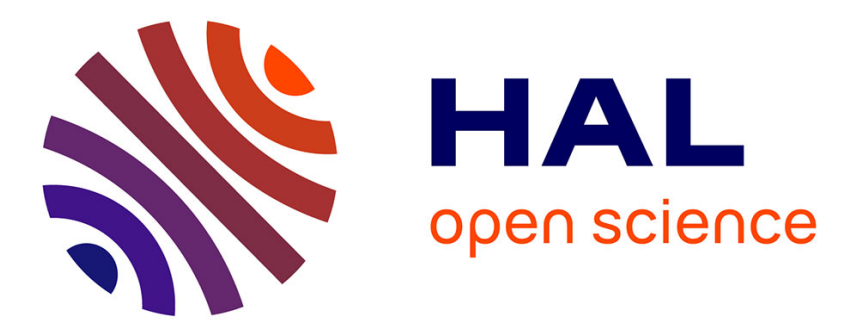

\title{
Evolution of cooperation under N-person snowdrift games
}

\author{
Max O. Souza, Jorge M. Pacheco, Francisco C. Santos
}

\section{To cite this version:}

Max O. Souza, Jorge M. Pacheco, Francisco C. Santos. Evolution of cooperation under N-person snowdrift games. Journal of Theoretical Biology, 2009, 260 (4), pp.581. 10.1016/j.jtbi.2009.07.010 . hal-00554636

\section{HAL Id: hal-00554636 \\ https://hal.science/hal-00554636}

Submitted on 11 Jan 2011

HAL is a multi-disciplinary open access archive for the deposit and dissemination of scientific research documents, whether they are published or not. The documents may come from teaching and research institutions in France or abroad, or from public or private research centers.
L'archive ouverte pluridisciplinaire HAL, est destinée au dépôt et à la diffusion de documents scientifiques de niveau recherche, publiés ou non, émanant des établissements d'enseignement et de recherche français ou étrangers, des laboratoires publics ou privés. 


\section{Author's Accepted Manuscript}

Evolution of cooperation under N-person snowdrift games

Max O. Souza, Jorge M. Pacheco, Francisco C. Santos

PII: S0022-5193(09)00316-6

DOI: doi:10.1016/j.jtbi.2009.07.010

Reference: YJTBI 5621

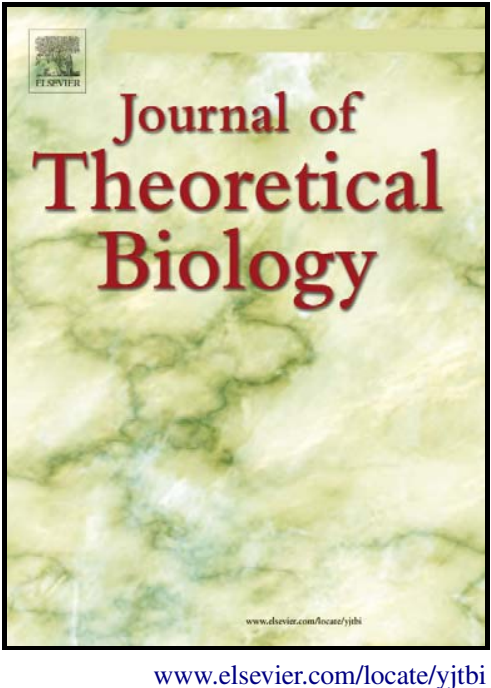

To appear in: $\quad$ Journal of Theoretical Biology

Received date: $\quad 6$ March 2009

Revised date: $\quad 3$ July 2009

Accepted date: 6 July 2009

Cite this article as: Max O. Souza, Jorge M. Pacheco and Francisco C. Santos, Evolution of cooperation under N-person snowdrift games, Journal of Theoretical Biology, doi:10.1016/j.jtbi.2009.07.010

This is a PDF file of an unedited manuscript that has been accepted for publication. As a service to our customers we are providing this early version of the manuscript. The manuscript will undergo copyediting, typesetting, and review of the resulting galley proof before it is published in its final citable form. Please note that during the production process errors may be discovered which could affect the content, and all legal disclaimers that apply to the journal pertain. 


\title{
Evolution of Cooperation under N-person Snowdrift Games
}

\author{
Max O. Souza \\ Departamento de Matemática Aplicada, Universidade Federal Fluminense, R. Mário Santos Braga, s/n, \\ Niterói RJ, 24020-140, Brasil \\ Jorge M. Pacheco \\ ATP-group/CFTC and Departamento de Física da Faculdade de Ciências, P-1649-003 Lisboa Codex, \\ Portugal \\ GADGET, Apartado 1329, 1009-001 Lisboa, Portugal \\ Francisco C. Santos \\ MLG and IRIDIA/CoDE, Université Libre de Bruxelles, Av. F. Roosevelt 50, CP 194/6, Brussels, Belgium \\ GADGET, Apartado 1329, 1009-001 Lisboa, Portugal
}

\begin{abstract}
In the animal world, performing a given task which is beneficial to an entire group requires the cooperation of several individuals of that group who often share the workload required to perform the task. The mathematical framework to study the dynamics of collective action is game theory. Here we study the evolutionary dynamics of cooperators and defectors in a population in which groups of individuals engage in N-person, non-excludable public goods games. We explore an N-person generalization of the well-known two-person Snowdrift Game. We discuss both the case of infinite and finite populations, taking explicitly into consideration the possible existence of a threshold above which collective action is materialized. Whereas in infinite populations, an Nperson Snowdrift Game (NSG) leads to a stable coexistence between cooperators and defectors, the introduction of a threshold leads to the appearance of a new interior fixed point associated with a coordination threshold. The fingerprints of the stable and unstable interior fixed points still affect the evolutionary dynamics in finite populations, despite evolution leading the population inexorably to a monomorphic end-state. However, when the group size and population size become comparable, we find that spite sets in, rendering cooperation unfeasible.
\end{abstract}

Key words: Evolutionary dynamics, evolutionary game theory, cooperation, public goods, co-existence 


\section{Introduction}

Evolutionary game theory has been widely employed in the study of the evolution of cooperation at different levels of organization (Axelrod and Hamilton, 1981; Maynard-Smith, 1982; Boyd and Richerson, 1985; Hofbauer and Sigmund, 1998; Skyrms, 2001; Macy and Flache, 2002; Hammerstein, 2003; Skyrms, 2004; Nowak and Sigmund, 2004; Santos and Pacheco, 2005; Santos et al., 2006; Ohtsuki et al., 2006; Nowak, 2006; Santos et al., 2008). Special attention has been paid to two-person dilemmas such as the Prisoners Dilemma (PD), the Snowdrift Game (SG) (Sugden, 1986) and the Stag-Hunt game (SH) (Skyrms, 2004), which constitute powerful metaphors to describe conflicting situations often encountered in the natural and social sciences (Macy and Flache, 2002; Skyrms, 2004). Many real-life situations, however, are associated with collective action based on joint decisions made by a group often involving more than 2 individuals. This is the case, for instance, in the upper primates, where problems of collective action are recurrent (Boyd and Richerson, 1985; Boehm, 1999). This type of problems are best dealt-with in the framework of N-person games (Hardin, 1968; Schelling, 1973; Dawes, 1980; Boyd and Richerson, 1988; Kollock, 1998; Hauert et al., 2006, 2007). Describing the evolutionary dynamics of many-person games provides a richer scenario of possibilities, as captured by the words of late W. D. Hamilton (Hamilton, 1975) in connection with three-person games:

"The theory of many person games may seem to stand to that of two-person games in the relation of sea-sickness to a headache."

Here we investigate the evolutionary dynamics of $\mathrm{N}$-person generalizations of the SG. In the standard SG, two individuals are driving on a road which is blocked by a snowdrift. To proceed with their journey home, the snow must be removed. Three possibilities occur: No-one shovels, and hence no-one gets home: The two drivers cooperate and shovel, and both get home, each one sharing the workload of shoveling the snow. If only one driver decides to shovel, both get home despite one driver incurring the entire cost of snow shoveling. If we define the benefit of getting home as $b$ and the cost of shoveling as $\mathrm{c}$, then if both drivers cooperate and shovel, each gets $b-c / 2$. If both defect, no one gets anything - 0 . If one cooperates and the other defects, the Cooperator (C) gets $b-c$ while the defector (D) gets $b$. Assuming, as usual, that the benefit is greater than the cost, we get a payoff ranking characteristic of a chicken, hawk-dove or snowdrift dilemma (Maynard-Smith, 1982). The generalization of this game to a public goods game involving $\mathrm{N}$ players is straightforward. To remain with the previous example, we can imagine that the snowdrift occurs at a cross-road where $N$ drivers meet. Again, all want to go home (getting all the same benefit $b$ ), but perhaps not all are willing to shovel. If all shovel, then each gets $b-c / N$. But if only $k$ individuals shovel (C), they get $b-c / k$ whereas those who defect by refusing to shovel get home 
for free and get $b$.

There are many examples in our everyday life and throughout our history, where instances of N-person snowdrift games (NSG) are or have been at stake (see below). Moreover, in some of these instances, it is often the case that no common benefit is produced unless its cost is shared by a minimum threshold of cooperating individuals. In keeping with the metaphor introduced above, the fact that individuals have a finite capacity of clearing the snow, combined with the risk that a new snowdrift may happen, further blocking the road, implies that a minimum threshold of people must cooperate (shovel) so that the road is cleared before the next snowdrift eventually happens.

The existence of thresholds in NSG abounds. For example, not all Amish need to participate in the construction of a church for the church to be built (Weir, 1985). Yet, the more contribute the better, since the effort to be invested by each member of the construction group will be smaller. On the other hand, the cost of building a church cannot be provided by a single individual. In this example, the public good is the church, so once it is built all get access to this non-excludable public-good. However, the more Amish help building the church, the less effort (cost) is required to each of them to produce the public good, given that the minimum threshold of individuals required to build the church is met. In addition, the size of the church, or the benefits of having one, do not necessarily increase with the number of individuals that worked on it.

Similarly, when steady and heavy rain signals the possibility of large floods, the set up of protection via sandbag levees requires the coordinated action of a minimum threshold of people for the flood to be prevented letting all enjoy the same benefit. Once this threshold is surpassed, the more people help, the less the individual cost of each of the contributors.

A feature of these examples which is common to all tasks of collective action captured in the framework of a NSG is the fact that a given cost is required for a benefit to be produced. Unlike a N-person Prisoners Dilemma (Bach et al., 2006; Pacheco et al., 2009), all individuals in the group get a benefit which does not depend on the number of contributors and on the size of the groups, to the extent that they manage to pay the necessary cost. In this case, the cost of producing the benefit is shared among all those who cooperate.

For a given group of size $N$, we define a threshold $1 \leq M \leq N$ such that only when the number $k$ of $\mathrm{Cs}$ in the group is at least $M(k \geq M)$ a public good is achieved. Since a cost $c$ is required to produce a benefit $b$, this means that, whenever $k \geq M$, each $\mathrm{C}$ pays a cost $c / k$. On the other hand, whenever $k<M$ no benefit is produced, despite each $\mathrm{C}$ "giving her best" to achieve such goal. Hence, the maximum cost each $\mathrm{C}$ may expend is $c / M$. Back to the church example, $M$ is the minimum number of equally 
apt individuals required to build a church. In the case of flood protection, $M$ equally capable individuals must be able to set up an artificial dam using sandbag levees in time before the water level surpasses the critical point. Clearly, the assumption that all individuals are equally capable of contributing to the public good is a simplification. Relaxing it will be deferred to future work.

In table 1 we summarize the payoffs of Cs and Ds in any case (as usual in N-person games, $k=0$ means no cost is expended and no benefit is produced).

Table 1: Payoff values for the N-person snowdrift game.

\begin{tabular}{|l||c|c|}
\hline Payoff obtained & $C$ & $D$ \\
\hline $1 \leq k<M$ & $-\frac{c}{M}$ & 0 \\
\hline$k \geq M$ & $b-\frac{c}{k}$ & $b$ \\
\hline
\end{tabular}

We shall assume a population of size $Z$, from which groups of size $N$ are randomly sampled. We shall first study the conventional limit in which $Z \rightarrow \infty$, under deterministic replicator dynamics. Subsequently, we shall consider stochastic dynamics in finite populations. The fitness of individuals is determined by their payoff collected when engaging in an $\mathrm{N}$-person $\mathrm{SG}$, requiring at least $0<M<N$ individuals to produce any public good at all. We shall find that requiring a minimum threshold of cooperators to produce a benefit leads to the appearance of coordination features in an otherwise coexistence game. Hence, we obtain a richer evolutionary dynamics scenario in infinite populations. We find that this scenario remains qualitatively valid whenever we remove the approximation of assuming infinite populations, although the stochastic dynamics only ends whenever a monomorphic population is reached. Nonetheless, and similar to what was found for the N-person Stag-Hunt game (Pacheco et al., 2009), for small populations and/or group sizes spanning nearly the entire population, we observe the "spite" effect first noted by Hamilton (Hamilton, 1970) which works against cooperation.

\section{Evolutionary dynamics of the N-person SG in infinite populations}

Let us assume a very large population $(Z \rightarrow \infty)$, a fraction $x$ of which is composed of Cs, the remaining fraction $(1-x)$ being Ds. Let groups of $\mathrm{N}$ individuals be sampled randomly from the population. Consequently, the average fitness of Ds in this population is given by 


$$
f_{D}=\sum_{k=0}^{N-1}\left(\begin{array}{c}
N-1 \\
k
\end{array}\right) x^{k}(1-x)^{N-1-k} \Pi_{D}(k)
$$

whereas the average fitness of $\mathrm{Cs}$ is given by

$$
f_{C}=\sum_{k=0}^{N-1}\left(\begin{array}{c}
N-1 \\
k
\end{array}\right) x^{k}(1-x)^{N-1-k} \Pi_{C}(k+1)
$$

Indeed, such random sampling leads to groups whose composition follows a binomial distribution. Individual payoffs $\Pi_{C}(k)$ and $\Pi_{D}(k)$ are listed in Table 1. Let us start by assuming that one individual can bear the cost $c$ of producing the public benefit $b$ $(M=1)$. From the replicator equation (Hofbauer and Sigmund, 1998)

$$
\dot{x}=x(1-x)\left(f_{C}-f_{D}\right)
$$

it follows that there exists an interior stable fixed point $x^{*}$, satisfying

$$
f_{C}\left(x^{*}\right)-f_{D}\left(x^{*}\right)=0
$$

where $x^{*}$ is the real solution of the equation (Zheng et al., 2007)

$$
\frac{c}{b}\left(1-x^{*}\right)^{N}+N x^{*}\left(1-x^{*}\right)^{N-1}-\frac{c}{b}=0
$$

which, for arbitrary group size N, can be solved numerically as shown in Fig. 1.

Similar to the 2-person game, the NSG provides conditions for the stable co-existence of Cs and Ds. As shown in Fig. 1, the equilibrium abundance of cooperators decreases with increasing group size $N$ and decreasing benefit-to-cost ratio $b / c$.

Let us now consider the case in which, only when $k \geq M \geq 1$, a public benefit $b$ will be available to all in the group. Similarly, to the $M=1$ case, collective benefit will be achieved whenever all contributions sum to $c$, i.e., when at least $M$ individuals contribute $c / M$. We may formally write the payoffs in Table 1 in the form

$$
\Pi_{D}(k)=b \theta(k-M)
$$

for the payoff of a defector in the group and

$$
\Pi_{C}(k)=\Pi_{D}(k)-\frac{c}{k} \theta(k-M)-\frac{c}{M}(1-\theta(k-M))
$$

for the payoff of a cooperator in the same group, where the Heaviside step function $\theta(x)$ satisfies $\theta(x<0)=0$ and $\theta(x \geq 0)=1$. Since the public good is only achieved when $k \geq M$, only in this case the total cost is shared among cooperative individuals. Otherwise, each individual contributes the most without succeeding in producing the 
required cost. In other words, cooperators may expend a cost in vain. Such scenario leads to a $\mathrm{SG}$ when $\mathrm{N}=2$ and $\mathrm{M}=1$, whereas $\mathrm{N}=\mathrm{M}=2$ leads to a $\mathrm{SH}$ dilemma (see below). Hence, we expect that introducing a threshold in the NSG may lead to some type of coordination dynamics.

Substituting Eqs. 6 and 7 into Eq. 1 and 2 leads to

$$
\begin{aligned}
f_{C}-f_{D}=\frac{c}{x N} & \left\{N \frac{b}{c}\left(\begin{array}{c}
N-1 \\
M-1
\end{array}\right) x^{M}(1-x)^{N-M}-\right. \\
- & {\left.\left[1+\sum_{k=0}^{M-1}\left(\begin{array}{l}
N \\
k
\end{array}\right) x^{k}(1-x)^{N-k}\left(\frac{k}{M}-1\right)\right]\right\} . }
\end{aligned}
$$

For $M=1$, we re-obtain equation (5). When $N=M=2$, we find that $x^{*}=\frac{c}{2 b}$ is an unstable fixed point, hence we have a $\mathrm{SH}$.

For $N>2$ and $M>1$ there is no analytic solution. Similar to the case when $M=1$, we can easily solve Eq. (8) numerically, as shown in Fig. 2. For most values of the benefit-to-cost ratio $b / c$, the existence of a threshold $M>1$ provides sufficient conditions for a completely new evolutionary dynamics. In addition to a stable fixed point also present in the $M=1$ case, there is an emerging new unstable equilibrium which divides the system into two well-defined basins of attraction (see below). Depending on the benefit-to-cost ratio $b / c$, the system will always end up either in full defection or in a equilibrium where cooperators and defectors co-exist. As shown in Fig. 2, the fraction of cooperators in the co-existence equilibrium increases with the value of the threshold $M$. This can be understood given that the individual cost of each cooperator decreases with increasing $M$. In addition, the relative size of each basin of attraction, defined by the position of the unstable equilibrium, reflects the difficulty to achieve collective coordination whenever $M>1$, creating distinct games and dynamics with different risk-dominant strategies. We can prove some general properties of Eq. (8) which warrant that the behavior depicted in Fig. 2 is always valid. More precisely, we prove in the appendix that there exists a critical value of the cost-to-benefit ratio $c / b$ - referred as $\bar{\gamma}$ below — and a critical fraction of cooperators $\bar{x}$, depending only on $N$ and $M$ such that

1. Whenever $\frac{c}{b}>\bar{\gamma}$ the evolutionary dynamics exhibits no interior fixed points;

2. Whenever $\frac{c}{b}=\bar{\gamma}$ there is only one interior fixed point, which we denote by $\bar{x}$, corresponding to an unstable fixed point;

3. Whenever $\frac{c}{b}<\bar{\gamma}$ there are two interior fixed points $x_{L}<\bar{x}<x_{R}$, such that $x_{L}$ is unstable and $x_{R}$ is stable. 
We are not able to provide an explicit expression for $\bar{x}$, except when $\epsilon=\frac{N-M}{N} \ll 1$, in which case we derive in the appendix the asymptotic approximation

$$
\bar{x}=\frac{M}{N}+\mathcal{O}\left(\epsilon^{N-M+1}\right) .
$$

In the main panel of Fig. 3 we show the accuracy of Eq. (9) in estimating the location of the critical fraction $\bar{x}$ for several combinations of the ratio $M / N$.

It is noteworthy that, in item 1 above, nothing prevents that $\bar{\gamma}>1$, which leads to situations in which cooperation is feasible even when $b<c$. Numerical results show that the critical benefit-to-cost ratio, for $1<M<N$, is a decreasing function of $M$ : For small $M$, the critical value satisfies $c<b$; however, for larger values of $M$, we observe that we might have a critical value with $c>b$, as illustrated in the inset of Fig. 3 (see also Fig. 2). The reason for this seems to be that, for large $M$, the individual effort required to maintain a collective benefit is spread more evenly among the participants, thus helping to promote cooperation. Indeed, the maximum individual contribution of cooperators is given by $c / M$, a decreasing function of $M$. On the other hand, as shown in Fig. 2, for larger $M$ the minimum coordination among cooperators $\left(x_{L}\right)$ necessary to produce such collective goal increases, thus reducing the chances of cooperators.

Fig. 2 shows results for a fixed group size of $N=20$ and variable threshold values of $M$ (main panel). For each value of $M$ there is a critical benefit-to-cost value $b / c$ above which two interior fixed points emerge. These can be found in Fig. 2 by drawing a horizontal line at a fixed $b / c$ - its intersection with the appropriate curve for a given threshold $M$ provides the location of the points. As shown in Fig. 2, one root corresponds to an unstable fixed point $\left(x_{L}\right)$ and the other to a stable fixed point $\left(x_{R}\right)$ inducing a coexistence between Cs and Ds (see appendix for a general proof). This means there is a range of values of $x\left(x_{L}<x<x_{R}\right)$, in which Cs are favored against Ds $\left(f_{C}(x)>f_{D}(x)\right)$. When $x>x_{L}$, the system will always evolve to the mixed configuration given by $x_{R}$, and below $x_{L}$ all individuals will end up refusing to contribute to the public good.

\section{Evolutionary dynamics in finite populations}

The evolutionary dynamics in large, finite populations, has been pioneered in economics by Young (Young, 1993) and by Kandori, Mailath and Rob (Kandori et al., 1993), in the limit where mutations are rare. Here we focus on a well-mixed population of finite size $Z$ in the absence of mutations. The fraction of cooperators $(k / Z)$ is no longer a continuous variable, varying in steps of $1 / Z$. Also, sampling of individuals is no longer binomial, following a hypergeometric distribution and can now be written 
as (Pacheco et al., 2009)

$$
f_{C}(k)=\left(\begin{array}{c}
Z-1 \\
N-1
\end{array}\right) \sum_{j=0}^{-1}\left(\begin{array}{c}
k-1 \\
j
\end{array}\right)\left(\begin{array}{c}
Z-k \\
N-j-1
\end{array}\right) \Pi_{C}(j+1)
$$

and

$$
f_{D}(k)=\left(\begin{array}{l}
Z-1 \\
N-1
\end{array}\right)^{-1} \sum_{j=0}^{N-1}\left(\begin{array}{l}
k \\
j
\end{array}\right)\left(\begin{array}{l}
Z-k-1 \\
N-j-1
\end{array}\right) \Pi_{D}(j)
$$

respectively, where we impose that the binomial coefficients satisfy $\left(\begin{array}{l}k \\ j\end{array}\right)=0$ if $k<0$.

We adopt a stochastic birth-death process (Karlin and Taylor, 1975) combined with the pairwise comparison rule (Traulsen et al., 2006, 2007a,b) in order to describe the evolutionary dynamics of Cs (and Ds) in a finite population. Under pairwise comparison, two individuals from the population, A and B are randomly selected for update (only the selection of mixed pairs can change the composition of the population). The strategy of A will replace that of B with a probability given by the Fermi function (from statistical physics)

$$
p=\frac{1}{1+e^{-\beta\left(f_{A}-f_{B}\right)}} .
$$

The reverse will happen with probability $1-p$. The quantity $\beta$, which in physics corresponds to an inverse temperature, controls the intensity of selection: For $\beta<<$ 1 selection is weak, and one recovers the replicator equation in the limit $Z \rightarrow \infty$ (Traulsen et al., 2006, 2007a,b). For arbitrary $\beta$, the quantity corresponding to the right hand side of the replicator equation, specifying the gradient of selection, is given in finite populations by (Traulsen et al., 2006, 2007a,b)

$$
g(k) \equiv T^{+}(k)-T^{-}(k)=\frac{k}{Z} \frac{Z-k}{Z} \tanh \left\{\frac{\beta}{2}\left[f_{C}(k)-f_{D}(k)\right]\right\}
$$

The right hand side of $g(k)$ is similar to the replicator equation, only that the (nonlinear) pairwise comparison (Traulsen et al., 2006, 2007a,b) defined in Eq. 12 leads to the appearance of the hyperbolic tangent of the fitness difference, instead of the fitness difference. This has implications in the characteristic evolutionary times, which now depend on $\beta$ (Traulsen et al., 2006, 2007a,b), but not in what concerns the roots of $g(k)$. Importantly, the evolutionary dynamics in finite populations will only stop whenever the population reaches a monomorphic state $(k=0$ or $k=Z)$. Hence, the sign of $g(k)$, which indicates the direction of selection, is important in that it may strongly influence the evolutionary time required to reach any of the absorbing states.

In Fig. 4, we show how the qualitative behavior of selection under stochastic dynamics in finite populations mimics closely that already encountered in the previous section (c.f. Fig. 2), associated with deterministic dynamics in infinite populations. Although the population will always fixate in one of the two absorbing states $(k=0$ and 
$k=Z$ in the absence of mutations), selection will act to drive the population toward a composition reflecting the rightmost root of $g(k)$, which constitutes the deepest point of the basin of attraction of the evolutionary dynamics.

On the other hand, as the group size approaches the population size the previous basin of attraction is reduced. In Fig. 5 we show a typical behavior of $g(k)$ as a function of the fraction of cooperators $k / Z$ for fixed population size $Z=50$, threshold $M=$ 5 and different group sizes $N$. As $N$ increases, cooperation becomes increasingly unfeasible - in the limit when $N \rightarrow Z$, cooperators have no chance and defectors dominate unconditionally. Moreover, for a given $b / c$ ratio, the existence of a finite population analogue of a stable root of $g(k)$ (in infinite populations) occurs for values of the frequency $k / Z$ of cooperators which decrease as $N$ increases (see inset of Fig. 5). This has been first noted by Hamilton (Hamilton, 1970) and reflects the occurrence of "spite" which works against cooperation, as illustrated in Fig. 4 and 5.

\section{Conclusion}

In this paper we considered Public Goods dilemmas in which collective action leads to a coexistence of cooperators and defectors in infinite populations. We extend such a $\mathrm{N}$-person snowdrift game to situations in which a minimum of coordinated collective action is required to achieve a public good. In infinite, well-mixed populations, the existence of a threshold opens the possibility for the appearance of an additional fixed point, creating a complex dynamics with two interior fixed points in the replicator equation. The one at lower frequency of cooperators is always an unstable fixed point, which determines a threshold for cooperative collective action and triggers the appearance of coordination effects in an otherwise co-existence evolutionary game. The other, at higher frequency of cooperators, is a stable fixed point, and hence determines the final frequency of cooperators in the population, assuming the coordination threshold is surpassed. For the same individual capacity, larger groups are capable of generating larger public benefits. For fixed benefit-to-cost ratio, increasing $M$ leads to evolutionary coexistence for increasing fractions of cooperators. On the other hand, as shown in Fig. 2, the coordination barrier also becomes more difficult to overcome.

Once the simplifying assumption of an infinite population size is abandoned, the evolutionary dynamics of the N-person snowdrift game shows, qualitatively, a behavior which is similar to that found in infinite populations in what concerns the direction of selection. However, the finite population size leads the evolutionary dynamics to stop only when the population is monomorphic. On the other hand, whenever the population size is small or the group size approaches the population size, cooperators are always disadvantageous, irrespective of the benefit-to-cost ratio of cooperation, in 
accord with the so-called "spite" effect first described by Hamilton. In this context, it is worth referring that in the Amish example provided in the introduction, the entire community is called to participate, as well as the entire community benefits from the public good. Consequently, our model predicts that spite will be effective here. Perhaps not surprisingly, community enforcement (not dealt with here) is often used to ensure collective action.

In the N-person Snowdrift Game addressed here, and in the presence of a threshold $M>1$ we assumed that, whenever the number of cooperators was $k<M$, each cooperator was contributing an individual cost $c / M$ which, when aggregated, would not be sufficient to produce a benefit. Qualitatively, however, nothing changes in the evolutionary dynamics of the population if, instead of $c / M$, cooperators contribute individually a cost $c / k$ - in fact, the algebra is considerably simplified - or if we state that individual contribution is just a fraction $1 / \alpha$ of the total $\operatorname{cost} c$.

Overall, the necessity of coordination in N-person snowdrift game dilemmas is shown to increase the equilibrium fraction of cooperators. However, this enhancement comes together with a strong dependence of the initial level of coordination, since coexistence between cooperators only emerges when a minimum number of cooperators is already present in the population. Moreover, our results re-inforce the idea (Kollock, 1998; Pacheco et al., 2009) that even minor differences in the nature of collective returns and/or costs can have a profound effect on the final outcome of cooperation. We believe that, in public good dilemmas, the existence of thresholds constitutes the rule, rather than the exception. Our results show that, as a result, the evolutionary dynamics is enriched, enhancing the beauty and complexity of N-person social dynamics.

\section{Acknowledgments}

This work was supported by FAPERJ Brazil (MOS), FNRS Belgium (FCS) and FCT Portugal (JMP). 


\section{Appendix}

\section{Equilibria results for $N$-person snowdrift game in infinite populations}

From Eq. 8, we have that the problem of determining interior roots of the replicator equation can be reduced to the study of the roots of the polynomial

$$
\begin{aligned}
p(x)=N & \left(\begin{array}{l}
N-1 \\
M-1
\end{array}\right) x^{M}(1-x)^{N-M} \frac{b}{c}- \\
& {\left[1+\sum_{k=0}^{M-1}\left(\begin{array}{l}
N \\
k
\end{array}\right) x^{k}(1-x)^{N-k}\left(\frac{k}{M}-1\right)\right] }
\end{aligned}
$$

Let $\gamma=c / b$. We find that it will be more appropriate to study

$$
\begin{aligned}
p(x, \gamma)=N & \left(\begin{array}{l}
N-1 \\
M-1
\end{array}\right) x^{M}(1-x)^{N-M}- \\
& \gamma\left[1+\sum_{k=0}^{M-1}\left(\begin{array}{l}
N \\
k
\end{array}\right) x^{k}(1-x)^{N-k}\left(\frac{k}{M}-1\right)\right]
\end{aligned}
$$

which has the same roots as $p(x)$, and where we made the dependence on $\gamma$ explicit. Notice also that $p(x, \gamma)$ implies the same dynamics for the Replicator that the one implied by $p(x)$ up to a time rescaling.

We then have the following result

Theorem 1. There exists $0<\bar{\gamma}$ and $0<\bar{x}<1$ such that, if

1. $\bar{\gamma} / \gamma<1$, then the evolutionary dynamics has no interior equilibria.

2. $\bar{\gamma} / \gamma=1$, then $\bar{x}$ is a unique interior equilibrium.

3. $\bar{\gamma} / \gamma>1$, then there are two interior equilibria $x_{L}<\bar{x}<x_{R}$. Moreover, $x_{L}$ is always an unstable equilibrium point, while $x_{R}$ is always a stable point.

In order to prove Theorem 1, it turns out that is more convenient to determine what $\gamma$ will render a given $x \in(0,1)$ an interior point, rather than determining what $x$ are equilibria for a given $\gamma$. The reason for this is that the introduction of a threshold breaks the combinatorial structure of the binomial sample, which precludes a more direct calculation as found, for instance, in Hauert et al. (2006).

Let us define

$$
\Gamma(x)=\left\{\begin{array}{lc}
0, & x=0, \\
N\left(\begin{array}{l}
N-1 \\
M-1
\end{array}\right) \frac{x^{M}(1-x)^{N-M}}{1+\sum_{k=0}^{M-1}\left(\begin{array}{l}
N \\
k
\end{array}\right) x^{k}(1-x)^{N-k}\left(\frac{k}{M}-1\right)}, & 0<x \leq 1 .
\end{array}\right.
$$

Then $\Gamma:[0,1] \rightarrow \mathbb{R}$ is continuous in $[0,1]$ and differentiable in $(0,1)$. Also, by solving for $\gamma$ the equation $p(x, \gamma)=0$, it is straightforward to verify that we have the identity

$$
p(x, \Gamma(x))=0 .
$$


As mentioned above, $\Gamma(x)$ is responsible for the existence of a cost-to-benefit ratio at which a given interior $x$ can become an equilibrium of the replicator dynamics. The critical value $\bar{x}$ corresponds to the first interior equilibrium which emerges when $c / b=\bar{\gamma}$ and which divides the unit interval into two pieces, in which the stable and unstable equilibrium remain confined whenever $c / b<\bar{\gamma}$. The thrust of the argument is to study the number of solutions of $\Gamma(x)=\gamma$, for a given $\gamma$, which then can be used to prove Theorem 1. In order to achieve our goal, we establish a series of results about $\Gamma$. In what follows, we shall assume $N>2$ and $1<M<N$.

Proposition 1. There is a unique $\bar{x} \in(0,1)$ such that $\Gamma^{\prime}(\bar{x})=0$. Such $\bar{x}$ will be the unique point of global maximum for $\Gamma$.

Proof. We compute $\Gamma^{\prime}$ and, after extensive manipulation, we find that

$$
\Gamma^{\prime}(x)=N\left(\begin{array}{l}
N-1 \\
M-1
\end{array}\right) x^{M-1}(1-x)^{N-M-1} G(x) / D^{2}
$$

where

$$
G(x)=M-N x-\frac{1}{M} \sum_{k=0}^{M-1}\left(\begin{array}{l}
N \\
k
\end{array}\right) x^{k}(1-x)^{N-k}(M-k)^{2}
$$

and

$$
D=1+\sum_{k=0}^{M-1}\left(\begin{array}{l}
N \\
k
\end{array}\right) x^{k}(1-x)^{N-k}\left(\frac{k}{M}-1\right)
$$

Since

$$
\sum_{k=0}^{M-1}\left(\begin{array}{l}
N \\
k
\end{array}\right) x^{k}(1-x)^{N-k}\left(\frac{k}{M}-1\right) \geq-\sum_{k=0}^{M-1}\left(\begin{array}{l}
N \\
k
\end{array}\right) x^{k}(1-x)^{N-k}>-1
$$

have that $D$ is never zero. Hence, it is easy to see that, in $(0,1)$, the sign of $\Gamma^{\prime}$ will be the same as that of $G$, the same being the case for the roots of $\Gamma^{\prime}$ and the roots of $G$. Now, $G(0)=0$ and an easy calculation shows that $G^{\prime}(0)$; hence $G$ is positive near zero. Also, we have that $G(1)=M-N<0$. So $G$ has at least on root in $(0,1)$, since it is continuous.

We now prove that this root is unique by showing that $G^{\prime \prime}$ is single signed

Let $z=x /(1-x)$ and

$$
S=\sum_{k=0}^{M-1}\left(\begin{array}{l}
N \\
k
\end{array}\right) z^{k}(M-k)^{2}
$$

Then, after some manipulation, we can write

$$
G^{\prime \prime}(x)=-\frac{1}{M}(1-x)^{N-4}\left[N(N-1) S-2(N-1) S_{z}+S_{z z}\right],
$$


where $S_{z}=\frac{\partial S}{\partial z}$ and $S_{z z}=\frac{\partial^{2} S}{\partial z^{2}}$. The expression inside the square brackets can be written as

$$
\begin{aligned}
& \frac{(M-2)^{2} N^{2}(N-1)^{2}}{2} z^{2}+(N-1) N(M-1)^{2}(N-2) z+ \\
& +\sum_{k=3}^{M-1}\left(\begin{array}{c}
N \\
k
\end{array}\right) z^{k}(M-k)^{2}[N(N-1)-2(N-1) k+k(k-1)] .
\end{aligned}
$$

It is straightforward to see that the quadratic and linear terms in $z$ are positive, since $N>2$. Finally, we observe that the last expression in brackets, interpreted as a polynomial in $k$ has the roots $k=N-1$ and $k=N$. Since $k<M-1<N-1$, it is positive. This implies that $G^{\prime \prime}(x)<0$. Hence $G$ has a unique root, $\bar{x}$. Since, as we have already shown, $G$ is positive near zero, we have that $\Gamma^{\prime}(x)>0$, for $0<x<\bar{x}$ and that $\Gamma^{\prime}(x)<0$, for $\bar{x}<x<1$. Hence, $\Gamma$ has a maximum at $\bar{x}$. Since it is unique, and positive, it is the global maximum.

Proposition 2. Let $\bar{\gamma}=\Gamma(\bar{x})$, with $\bar{x}$ given above. Then the equation $\Gamma(x)=\gamma$ has

1. two solutions, $x_{L}$ and $x_{R}$, for $\gamma<\bar{\gamma}$. Moreover $x_{L} \in[0, \bar{x})$ and $x_{R} \in(\bar{x}, 1]$.

2. one solution for $\gamma=\bar{\gamma}$;

3. no solution for $\gamma>\bar{\gamma}$.

Proof. The cases $\gamma>\bar{\gamma}$ and $\gamma=\bar{\gamma}$ are immediate. For the remaining case note that, for $x \in[0, \bar{x})$, we have $\Gamma^{\prime}(x)>0$. Thus $\Gamma$ is monotonic increasing in $[0, \bar{x})$ and hence there is a unique $x_{L} \in[0, \bar{x})$ such that $\Gamma\left(x_{L}\right)=\gamma<\bar{\gamma}$. The argument for $x \in(\bar{x}, 1]$ is analogous, the only change being that $\Gamma$ is now monotonic decreasing in $(\bar{x}, 1]$.

Proof of Theorem 1. As for the number of equilibria, we observe that a pair $(x, \gamma)$ satisfies $p(x, \gamma)=0$ if, and only if, $\Gamma(x)=\gamma$. Hence, there is a bijection between the solution sets of each problem, and the results follows from Proposition 2.

Concerning the stability of the fixed points, when $\gamma<\bar{\gamma}$, let us write identity (15) as:

$$
N\left(\begin{array}{l}
N-1 \\
M-1
\end{array}\right)\left(\frac{x}{1-x}\right)^{M}-\Gamma(x)\left(1+\sum_{k=0}^{M-1}\left(\begin{array}{l}
N \\
k
\end{array}\right) x^{k}(1-x)^{N-k}\left(\frac{k}{M}-1\right)\right)=0 .
$$

Differentiating the above identity, without expanding, yields

$$
\begin{aligned}
& \left(N\left(\begin{array}{l}
N-1 \\
M-1
\end{array}\right)\left(\frac{x}{1-x}\right)^{M}\right)^{\prime}-\Gamma(x)\left(1+\sum_{k=0}^{M-1}\left(\begin{array}{l}
N \\
k
\end{array}\right) x^{k}(1-x)^{N-k}\left(\frac{k}{M}-1\right)\right)^{\prime}= \\
& =\Gamma^{\prime}(x)\left(1+\sum_{k=0}^{M-1}\left(\begin{array}{l}
N \\
k
\end{array}\right) x^{k}(1-x)^{N-k}\left(\frac{k}{M}-1\right)\right)
\end{aligned}
$$


which can be written, using the notation set in Proposition 1, as

$$
p^{\prime}(x, \Gamma(x))=\Gamma^{\prime}(x) D
$$

Thus, at a root, $p^{\prime}(x)$ has the same sign as the right hand side, which is the sign of $\Gamma^{\prime}(x)$, since $D>0$. We have already seen that $\Gamma^{\prime}\left(x_{L}\right)>0$ and $\Gamma^{\prime}\left(x_{R}\right)<0$. Thus, $x_{L}$ is an unstable point and $x_{R}$ is a stable point.

Let us now address the case $\gamma=\bar{\gamma}$. We must have

$$
\lim _{\gamma \rightarrow \bar{\gamma}^{-}} x_{L}=\bar{x} \quad \text { and } \lim _{\gamma \rightarrow \bar{\gamma}^{-}} x_{R}=\bar{x},
$$

with $x_{L}<x_{R}$, for $\gamma<\bar{\gamma}$. Also, as a consequence of the stability results for $\gamma<\bar{\gamma}$, we have that $p(x)<0$ for $x<x_{L}$, and $p(x)<0$ for $x>x_{R}$. Thus, at $\gamma=\bar{\gamma}$, we must have that $\bar{x}$ is a maximum of $p$ and, hence that $p(x) \leq 0$. Therefore, $\bar{x}$ is an unstable point.

Finally, we present a result concerning the location of $\bar{x}$.

Proposition 3. Let $x_{0}=\frac{M}{N}$ and assume that

$$
0<\epsilon=\frac{N-M}{N} \ll 1
$$

Then, we have that

$$
\bar{x}=x_{0}-\frac{x_{0}^{M}}{M}\left(\begin{array}{c}
N \\
M-1
\end{array}\right) \epsilon^{N-M+1}+\mathcal{O}\left(\epsilon^{N-M+2}\right) .
$$

Proof. Recall that $\bar{x}$ satisfies $G(\bar{x})=0$.We rewrite $G(\bar{x})=0$ as

$$
x_{0}-\bar{x}-\left(\epsilon+x_{0}-\bar{x}\right)^{N} M^{-1} \sum_{k=0}^{M-1}\left(\begin{array}{l}
N \\
k
\end{array}\right)\left(\frac{\bar{x}}{\epsilon+x_{0}-\bar{x}}\right)^{k}(M-k)^{2}=0 .
$$

From the proof of Proposition 1, we know that $G^{\prime}(\bar{x}) \neq 0$. Since $G$ is smooth, the Implicit Function theorem implies that $\bar{x}=\bar{x}(\epsilon)$ is smooth. Hence, we can find its Taylor expansion by formal substitution. By simple scaling arguments, we find that

$$
\bar{x}=x_{0}+x_{1} \epsilon^{N-M+1}+\mathcal{O}\left(\epsilon^{N-M+2}\right)
$$

By formal substitution, we then find

$$
x_{1}=-\frac{x_{0}^{M}}{M}\left(\begin{array}{c}
N \\
M-1
\end{array}\right) \text {. }
$$




\section{References}

Axelrod, R., Hamilton, W. D., 1981. The evolution of cooperation, Science 211, 13901396.

Bach, L. A., Helvik, T., Christiansen, F. B., 2006. The evolution of n-player cooperation - threshold games and ESS bifurcations J Theor. Biol. 238, 426-434.

Boehm, C., 1999. Hierarchy in the Forest: The Evolution of Egalitarian Behavior, (Harvard University Press).

Boyd, R. \& Richerson, P. J., 1988. The evolution of reciprocity in sizable groups. J Theor. Biol. 132 (2), 337-56.

Boyd, R. \& Richerson, P. J., 1985. Culture and the evolutionary process, (University of Chicago Press, Chicago, IL).

Dawes, R. M., 1980. Social dilemmas. Annual Review of Psychology 31, 169-193.

Hamilton, W., 1970. Selfish and spiteful behaviour in an evolutionary model. Nature 228, 1218-1220.

Hamilton, W., 1975. In: Biosocial anthropology, (Malaby Press, London), pp. 133155.

Hammerstein, P. (Ed.), 2003. Genetic and Cultural Evolution of Cooperation (MIT, Cambridge, MA).

Hauert, C., Michor, F., Nowak, M., Doebeli, M., 2006. The Tragedy of the Commons Science 162 (5364), 1243-8.

Hauert, C., Michor, F., Nowak, M., Doebeli, M., 2006. Synergy and discounting of cooperation in social dilemmas. J Theor. Biol. 239, 195.

Hauert, C., Traulsen, A., Brandt, H., Nowak, M., Sigmund, K., 2007. Via freedom to coercion: The emergence of costly punishment. Science 316, 1905-1907.

Hofbauer, J., Sigmund, K., 1998. Evolutionary Games and Population Dynamics (Cambridge Univ. Press, Cambridge, UK).

Kandori, M., Mailath, G., Rob, R. , 1993. Learning, mutation, and long-run equilibria in games. Econometrica 61, 29-56.

Karlin, S., Taylor, H., 1975. A first course in Stochastic Processes (2nd edition, Academic, London). 
Kollock, P.,1998. Social dilemmas: the anatomy of cooperation Annu. Rev. Sociol. 24, $183-214$.

Macy, M., Flache, A., 2002. Learning dynamics in social dilemmas. Proc Natl Acad Sci U S A 99, 7229-36-.

Maynard-Smith, J., 1982. Evolution and the Theory of Games (Cambridge University Press, Cambridge).

Nowak, M., 2006. Five rules for the evolution of cooperation. Science 314, 1560-1563.

Nowak, M., Sigmund, K., 2004. Evolutionary dynamics of biological games. Science 303, 793-799.

Ohtsuki, H., Hauert, C., Lieberman, E., Nowak, M., 2006. A simple rule for the evolution of cooperation on graphs and social networks. Nature 441, 502-505.

Pacheco, J. M., Santos, F. C., Souza, M. O., Skyrms, B., 2009. Evolutionary dynamics of collective action in n-person stag-hunt dilemmas. Proc. Royal. Soc. B 276 (1655):315-21.

Santos, F., Pacheco, J. M., 2005. Scale-free networks provide a unifying framework for the emergence of cooperation. Phys. Rev. Lett. 95, 098104.

Santos, F., Pacheco, J. M., Lenaerts, T., 2006. Evolutionary dynamics of social dilemmas in structured heterogeneous populations. Proc Natl Acad Sci U S A 103, 34903494.

Santos, F., Santos, M., Pacheco, J. M., 2008. Social diversity promotes the emergence of cooperation in public goods games. Nature 454, 213-216.

Schelling,T. C.,1973. Hockey Helmets, Concealed Weapons, and Daylight Saving: A Study of Binary Choices With Externalities J. Conflict Resolution 17, 381.

Skyrms, B., 2001. The stag hunt. Proceedings and Addresses of the American Philosophical Association 75, 31-41.

Skyrms, B., 2004. The Stag Hunt and the Evolution of Social Structure. (Cambridge University Press).

Sugden, R., 1986. The economics of rights, co-operation and welfare. (Basil Blackell, Oxford, UK).

Traulsen, A., Nowak, M., Pacheco, J. M., 2006. Stochastic dynamics of invasion and fixation. Phys. Rev. E 74, 011909. 
Traulsen, A., Nowak, M., Pacheco, J. M., 2007a. Stochastic payoff evaluation increases the temperature of selection. J. Theor. Biol. 244, 349-356.

Traulsen, A., Pacheco, J. M., Nowak, M., 2007b. Pairwise comparison and selection temperature in evolutionary game dynamics. J. Theor. Biol. 246, 522-529.

Weir, P., 1985. Witness. (Paramount Pictures, USA)

Young, H., 1993. The evolution of conventions. Econometrica 61, 57-84.

Zheng, D., Yin, H., Chan, C., Hui, P., 2007. Cooperation behavior in a model of evolutionary snowdrift games with n-person interactions. Europhysics Letters 8018002 18006. 

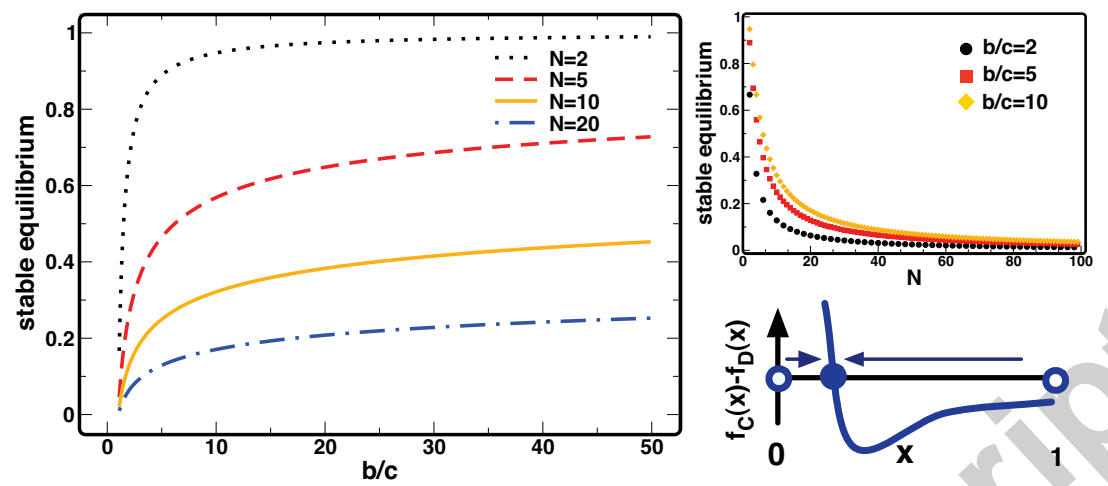

Figure 1: Stable equilibria of the N-person SG in infinite populations. In the main panel we show the location of the stable interior equilibrium of the replicator dynamics equation as a function of the benefit-to-cost ratio $b / c$ for group sizes $\mathrm{N}=2$ (dotted black line), 5 (dashed red line), 10 (solid yellow line) and 20 (dash-dot blue line). The stable nature of this interior fixed point is illustrated in the inset on the lower right. In the small panel in the upper right we show how the location of the interior stable fixed point scales with group size for the three different benefit-to-cost ratios $b / c$ indicated. 

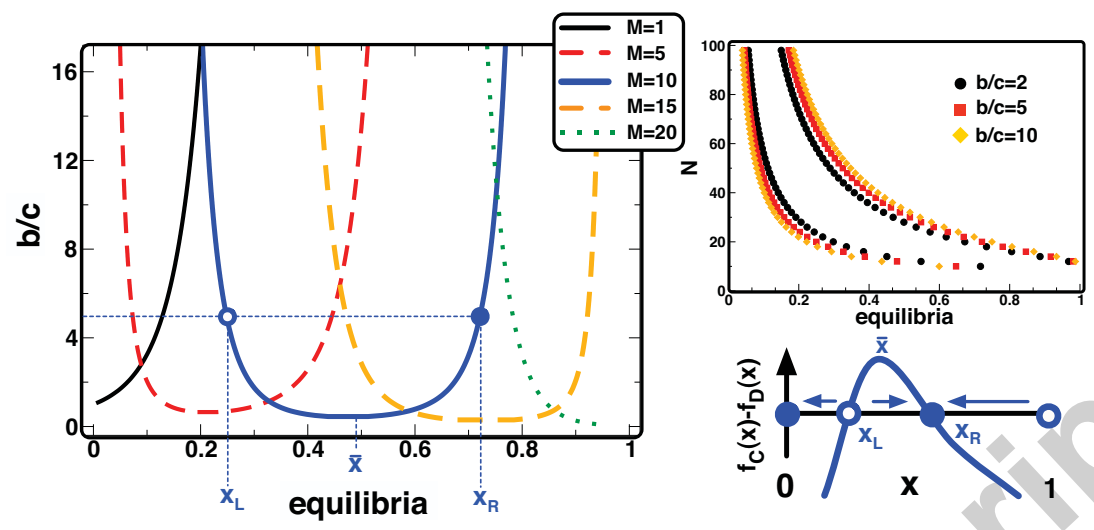

Figure 2: Equilibria of the N-person Snowdrift Game with threshold. We assume infinite, well-mixed populations, fix the group size at $N=20$ and vary the threshold $M$ above which cooperation leads to a common benefit $b$. The total cost involved is $c$. In the main panel we show how the occurrence of a threshold leads to the appearance of at most 2 interior fixed points $x_{L}$ and $x_{R}$, which can be found via the intersection of a horizontal line with the appropriate curve (illustrated for $M=10$ ); in this case, the leftmost root is always an unstable fixed point whereas the rightmost corresponds to stable fixed point, as illustrated in the small panel in the lower right (see main text for details). For a given $M / N$, there is a critical value $\bar{\gamma}$ for the critical cost-to-benefit ratio $c / b$ below which the 2 interior roots discussed above always exist. In the small panel (upper right) we show how these interior fixed points scale with variable group size $N$ for some values of the $b / c$ ratio indicated. For $\bar{\gamma} b<c$ no interior fixed points exist and defectors dominate unconditionally, whereas for $\bar{\gamma} b=c$ the only root corresponds to an unstable fixed point; it is noteworthy that $\bar{\gamma}$ increases with increasing $M / N$ (main panel) and can actually become larger than 1 (see main text for details). 


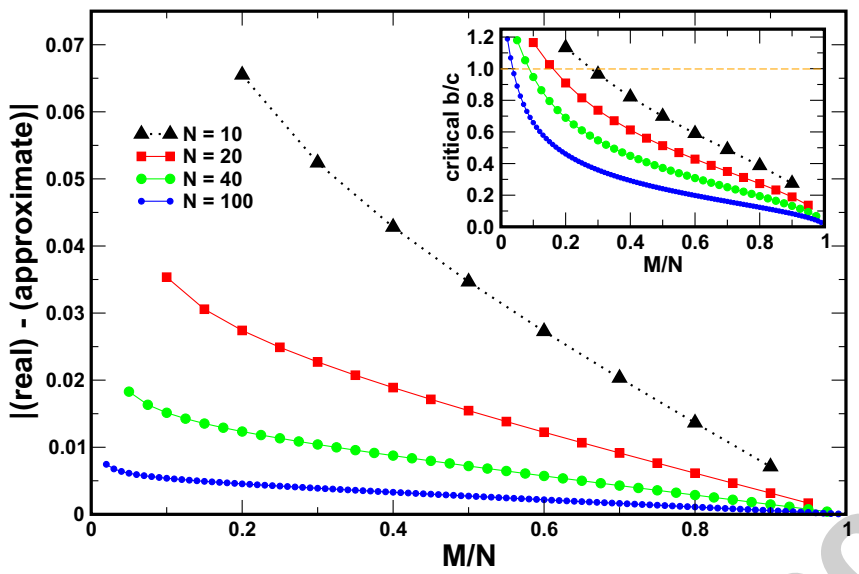

Figure 3: In the main panel we compare the numerical solution of $\bar{x}$ with the asymptotic approximation in Eq. (9), for different values of $M / N$ and group sizes $N=10,20,40$ and 100. In the inset we plot the critical value of $b / c$ as a function of $M / N$ for the same group sizes; for small $M / N$, the critical value behaves conventionally in the sense that $b>c$, cooperation being feasible only when the benefit outweighs the cost. With increasing $M / N$, however, we observe a decrease of the critical benefit-to-cost ratio $b / c$ (see main text for details). 


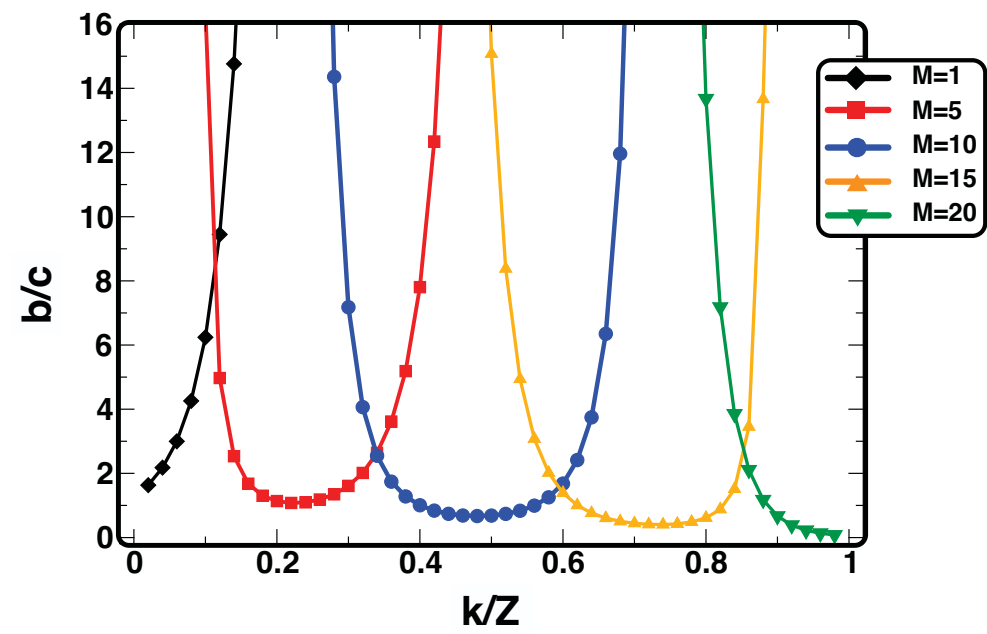

Figure 4: Equilibria of the N-person snowdrift game with threshold in finite populations. Population size is $Z=50$ and group size is $N=20$. We vary the threshold $M$ above which cooperation leads to a common benefit $b$. For each $k / Z$ we show the corresponding $b / c$ at which $g(k)=0$ (cf. Eq. (13)). Whenever the population size is large compared to group size, selection in finite populations is qualitatively similar to that in infinite populations. 


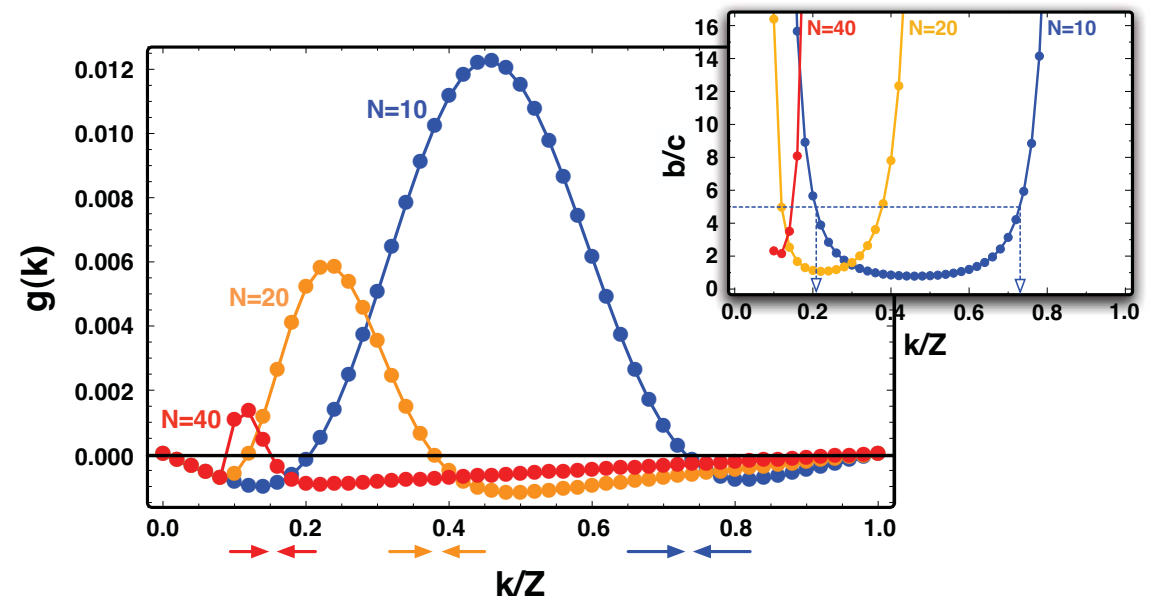

Figure 5: Effect of group size in the evolution of cooperation. In the main panel, we plot $g(k)$ (defined in Eq. (13)) as a function of the fraction of cooperators $k / Z$, for $b / c=5$. We fixed the population size at $Z=50$ and the threshold at $M=5$, while varying the group size $N$. As the group size approaches the population size, the range of values of $k / Z$ for which cooperation is advantageous $(g(k)>0)$ is reduced. In the inset, we show how the roots of $g(k)$ vary with $b / c$ for different values of the group size $N$. For $N \sim Z$ the roots of $g(k)$ disappear and defectors are always advantageous. 